\title{
The role of salvage surgery in the treatment of a gefitinib-resistant non-small cell lung cancer patient: a case report
}

\author{
Jing Zang ${ }^{1,2}$, Hidehito Horinouchi ${ }^{3}$, Jun Hanaoka $^{4}$, Kazuhito Funai $^{5}$, Noriaki Sakakura $^{6}$, Hu Liao $^{7}$ \\ ${ }^{1}$ Department of Ultrasound, West China Second University Hospital, Sichuan University/West China Women's and Children's Hospital, Chengdu, \\ China; ${ }^{2}$ Key Laboratory of Birth Defects and Related Diseases of Women and Children (Sichuan University), Ministry of Education, Chengdu, \\ China; ${ }^{3}$ Department of Thoracic Oncology, National Cancer Center Hospital, Chuo-ku, Tokyo, Japan; ${ }^{4}$ Division of General Thoracic Surgery, \\ Department of Surgery, Shiga University of Medical Science, Tsukinowacho, Seta, Otsu, Shiga, Japan; ${ }^{5}$ First Department of Surgery, Hamamatsu \\ University School of Medicine, Higashi-ku, Hamamatsu, Shizuoka Japan; ${ }^{6}$ Department of Thoracic Surgery, Aichi Cancer Center Hospital, \\ Chikusa-ku, Nagoya, Aichi, Japan; ${ }^{7}$ Department of Thoracic Surgery, West China Hospital, Sichuan University, Chengdu, China \\ Correspondence to: Hu Liao. Department of Thoracic Surgery, West China Hospital, Sichuan University, No. 37 Guoxue Alley, Chengdu, China. \\ Email: liaotiger_198653@163.com.
}

Submitted Jan 28, 2021. Accepted for publication May 28, 2021.

doi: $10.21037 /$ jtd-21-171

View this article at: https://dx.doi.org/10.21037/jtd-21-171

\section{Introduction}

Studies have revealed that the epidermal growth factor receptor (EGFR) mutation is one of the driving mutations in non-small cell lung cancer (NSCLC) $(1,2)$. Thus, EGFR tyrosine kinase inhibitors (TKIs) gradually have become one of the most widely used targeted therapies for advanced NSCLC patients $(3,4)$. Compared with standard adjuvant chemotherapy, gefitinib is considered to improve the progression-free survival (PFS) for NSCLC patients harbouring EGFR mutations (5).

However, approximately $65 \%$ of EGFR-TKI-sensitive NSCLC patients eventually developed into acquired resistance after 9-13 months of treatment (6). The mechanisms for EGFR-TKI resistance are controversial, Xiang and colleagues found that EGFR sensitive mutation and KRAS mutation might coexistence which triggered EGFR-TKI resistance (7). If patients develop gefitinib resistance, the best choice among subsequent treatment options still remains unclear. We present one case of salvage resection in the treatment of a gefitinib-resistant NSCLC patient. We present the following article in accordance with the CARE reporting checklist (available at https://dx.doi. org/10.21037/jtd-21-171).

\section{Case presentation}

A 49-year-old woman was diagnosed with stage IB adenocarcinoma (Figure 1A) after open right middle lobe (RML) resection and systematic lymph node dissection in 2011. She received four cycles of adjuvant Navelbine and cisplatin chemotherapy after surgery. Four years after surgery, the patient was found to have ipsilateral lung metastasis (Figure 1B). Gene detection of specimen revealed an exon 21 Leu858Arg substitution (L858R), and an oral gefitinib targeted therapy was applied to the patient. Three months after target therapy, chest computed tomography (CT) scan showed that the metastatic lesion was significantly reduced (Figure 1C), and a 3-month interval CT scan follow-up showed the lesion was in a stable condition. Twenty-seven months after target therapy, the metastatic lesion began to grow (Figure 1D), which was considered as a sign of resistance to gefitinib. After multiple disciplinary team (MDT) discussion, the patient received salvage surgery of video-assisted thoracoscopic surgery (VATS) wedge resection of the metastatic lesion. The postoperative pathological result and gene analysis indicated adenocarcinoma (Figure 2) with EGFR L858R and T790M mutations. However, osimertinib was not available in China 

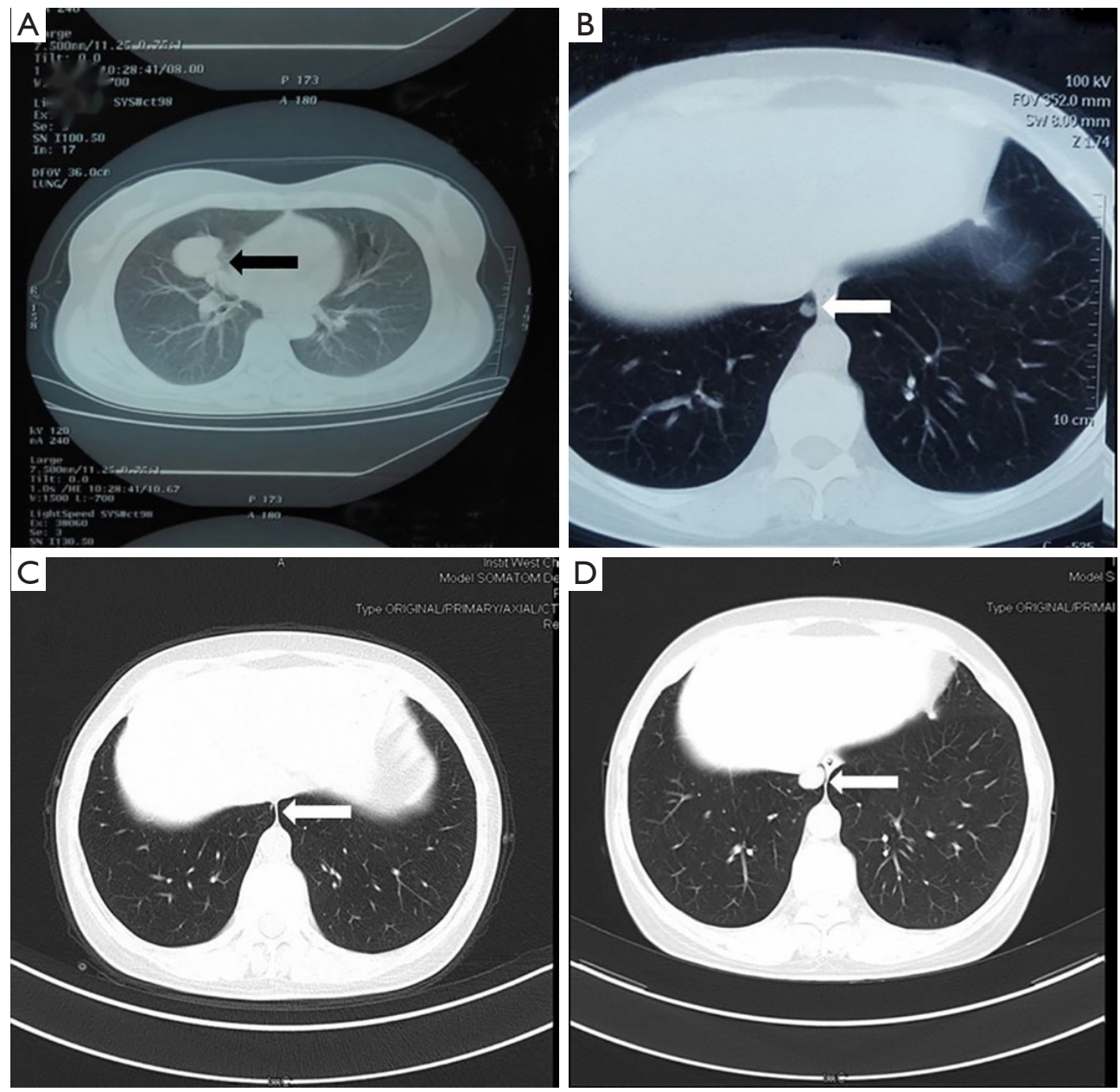

Figure $1 \mathrm{CT}$ scan of the primary lesion (black arrow) and metastatic lesion (white arrow). (A) A 4-cm mass in the right middle lobe. (B) Right lower lobe metastasis after surgery and adjuvant chemotherapy. (C) Three months after gefitinib therapy, the metastatic lesion was significantly reduced. (D) The metastatic lesion began to grow again, and drug resistance was clinically confirmed.

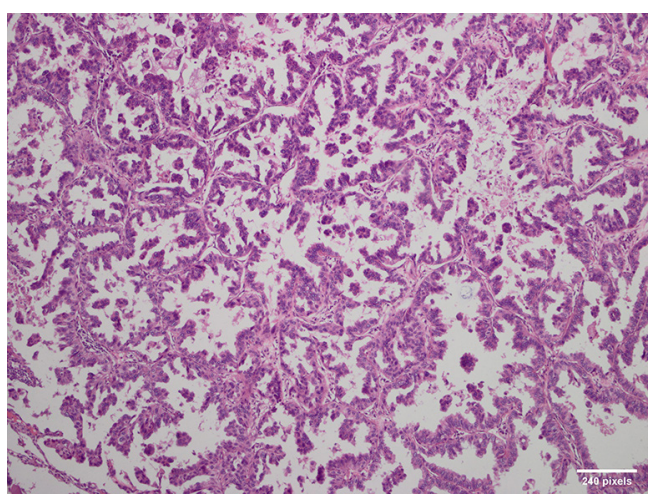

Figure 2 Pathological findings indicate a metastatic lesion along with papillary and micropapillary adenocarcinoma (HE staining; magnification $\times 100$ ). at that time, and the patient refused chemotherapy. MDT discussion suggested that the patient should continue gefitinib maintenance therapy after surgery. The patient developed into brain metastasis 10 months after surgery, and osimertinib combined with stereotactic radiosurgery was applied. Twelve months after the combined therapy, the patient was found to have bone and liver metastasis, along with progression of the brain metastatic lesion. Though the patient was given the best supportive care, she died of multiple organ dysfunction 9 months later. Written informed consent was obtained from the patient's family members for publication of this case report and all the affiliated images. All procedures performed in studies involving human participants were in accordance with 
the ethical standards of the institutional and/or national research committee(s) and with the Helsinki Declaration (as revised in 2013).

\section{IMDT discussion}

\section{Department of Oncology}

When compared with standard chemotherapy, the firstgeneration EGFR-TKIs have proven to be an effective first-line treatment $(8,9)$, which can increase the tumor response rate (10). Notably, in a number of studies, it was confirmed that EGFR-TKIs could offer a significant improvement in chemotherapy $(9,11,12)$. This firmly supports the wide application of targeted therapy for initial treatment for EGFR-mutated NSCLC patients. There is also evidence indicating that EGFR-TKIs involve a lower frequency of a serious adverse events (13). In this case, postoperative adjuvant chemotherapy yielded 4 years of PFS, and after progression, gefitinib successfully controlled the development of the disease and provided another 27 months of PFS. Recent studies have suggested that N1 and N2 EGFR-mutated NSCLC patients might benefit from postoperative adjuvant target therapy (14). However, in the early stage of EGFR-mutated NSCLC patients, first-line adjuvant EGFR-TKI target therapy after surgery should be applied with care.

\section{Department of Thoracic Surgery}

Osimertinib is widely accepted as a standard treatment for T790M-mutated gefitinib-resistant NSCLC. In this rare case, although the T790M mutation was detected after the resection of the metastasis, the patient did not receive osimertinib as the drug was not available at that time. This provided us an opportunity to observe the effect of surgery in the treatment of TKI-resistant NSCLC. Salvage resection offered the patient a 10-month PFS. Furthermore, the resected specimen allowed us to more clearly and deeply explore the mechanism of resistance. For resectable EGFRTKI-resistant NSCLC patients, salvage surgery may be an option after MDT discussion. However, there is scarce evidence for the efficiency of the surgery after EGFR-TKI resistance is confirmed. Hence, further research is needed to clarify this issue.

\section{Further issues for discussion regarding the diagnosis and treatment of this patient}

Question 1: In this case, due to the mutation detected, should targeted therapy have been applied right after her first surgery instead of chemotherapy?

Expert Opinion 1: Dr. Hidebito Horinouchi

Yes, I agree to continue gefitinib after salvage surgery. There are two main reasons which should be considered. First, although T790M resistant mutation was detected in surgical resected specimen, gefitinib still suppressed other lesions and could offer the patient further PFS after surgery. Second, chemotherapy could be applied even after emergence of other gefitinib resistant lesions if the patient observed cautiously.

\section{Expert Opinion 2: Dr. Fun Hanaoka}

Here, considering the primary site, clinical stage, and age, postoperative adjuvant therapy is recommended. However, as reported by BR.19, the safety and effectiveness of molecular-targeting agents for postoperative adjuvant chemotherapy have not yet been established; hence, treatment with cytotoxic anticancer agents is recommended. Molecular targeted drugs have excellent therapeutic effects, and become key drugs for post-recurrence therapy. If there is no difference in prognosis even if treatment is started after recurrence, and complete resection is achieved, adjuvant treatment with molecular-targeting agents is considered unnecessary. However, for disease-free survival (DFS), post-operative administration of erlotinib or gefitinib was reported to be useful. Furthermore, favorable effects of osimertinib were reported in American Society of Clinical Oncology conference of 2020. Based on these reports, there is a high possibility of moleculartargeting therapeutic agents being also used for adjuvant chemotherapy after surgery.

\section{Expert Opinion 3: Dr. Kazubito Funai}

No. I believe that targeted therapy should not have been performed right after her first surgery. Raphael et al. showed that adjuvant EGFR-TKI therapy extends the DFS in patients with EGFR-mutant NSCLC but does not improve the overall survival (OS) in their systematic review and meta-analysis (15). Therefore, the use of adjuvant TKIs is not recommended due to the lack of OS benefits. In fact, the use of an EGFR-TKI in postoperative adjuvant chemotherapy is not recommended by the Japanese 
guidelines.

\section{Expert Opinion 4: Dr. Noriaki Sakakura}

The final follow-up data of the CTONG1104 trial that was shown in ASCO 2020 demonstrated that patients with resected pN1-N2 NSCLC could obtain longer DFS and similar OS after postoperative gefitinib administration, compared with the outcomes after standard platinum-based chemotherapy in an adjuvant setting. Furthermore, the ADAURA trail currently suggests that adjuvant osimertinib can provide effective treatment for patients with EGFRmutated stage IB, II and IIIA NSCLC after complete resection. In the future, there will be more data showing the value of postoperative TKIs. For this case, it seems that a reasonable choice was made at that time.

\section{Question 2: After gefitinib resistance was evident following salvage surgery, should a later generation EGFR-TKI have been immediately applied? Expert Opinion 1: Dr. Hidehito Horinouchi}

Yes, I do use osimertinib at the point the patient developed definite progression after gefitinib as maintenance therapy following salvage surgery.

\section{Expert Opinion 2: Dr. Fun Hanaoka}

Later generation EGFR-TKI should not necessarily apply immediately. Here, considering the possibility of localized acquired resistance, restart of Gefitinib maintenance therapy as in this case is acceptable after complete resection of the recurrent site. With close follow-up, if there is a sign of recurrence, it is possible to switch treatment based on the mechanism of resistance detected.

\section{Expert Opinion 3: Dr. Kazubito Funai}

If the T790M mutation is positive, I give the patient Osimertinib immediately, as Osimertinib had a significantly greater effect than platinum-Pemetrexed in chemotherapy patients with T790M-positive advanced NSCLC in whom the disease had progressed during first-line EGFR-TKI therapy (16).

\section{Expert Opinion 4: Dr. Noriaki Sakakura}

This depends on whether other small metastatic lesions were present at the time of partial resection of the right lower lobe. In other words, that depends on whether there were other lesions that were responsive to gefitinib. If there were such lesions, gefitinib should have been continued. In the absence of such other lesions, osimertinib would probably be the better option to use. However, at that time, standard chemotherapy was a possible option.
Question 3: Is salvage surgery suitable for all the patients who develop targeted therapy resistance, or are there selection criteria for this kind of patient? Expert Opinion 1: Dr. Hidebito Horinouchi

No, I don't think salvage surgery for all EGFR-mutant patients who develop resistance. I agree that salvage surgery as well as other local ablative therapies are options even in patients with advanced NSCLC. Salvage surgery and local ablative therapies should be considered in such patients whose expected prognosis is apparently shorter than that of systemic therapies. For example, if patient developed 1st and 2nd generation EGFR-TKI resistance with T790M additional mutation, osimertinib should be the first choice rather than local therapies.

\section{Expert Opinion 2: Dr. Fun Hanaoka}

Patients with indication to surgery can be considered as candidates for salvage surgery, and development of treatment strategies for the recurrence type can be contemplated. However, if the possibility of recurrence after surgery is high, chemotherapy should be preferred over surgery, especially where effective drugs for gene mutations are known and available. If salvage surgery is performed, cases wherein postoperative activities of daily living (ADL) may be significantly reduced or continuation of chemotherapy may be difficult to tolerate should be avoided. Complete resection is the best treatment modality; however, if the treatment of recurrence site is possible by combining with radiation therapy, tumor reduction by surgery is also acceptable.

\section{Expert Opinion 3: Dr. Kazubito Funai}

I do not believe that salvage surgery is suitable for all patients who develop targeted therapy resistance. In cases with solitary lung metastasis, as in this case, salvage surgery may be indicated for differentiating metachronous primary lung cancer. There is the additional advantage of T790M mutations being able to be confirmed by salvage surgery.

Although there is no clear evidence, I personally think that salvage surgery for resectable patients who develop targeted therapy resistance could be an option. However, even in such cases, I feel that systemic treatment is essential.

We previously reported on "Surgical resection after gefitinib treatment in patients with lung adenocarcinoma harboring epidermal growth factor receptor gene mutation" (17). This patient is currently alive at 160 months after surgery without recurrent lesions. 
Expert Opinion 4: Dr. Noriaki Sakakura

At present, the indications for local treatment of oligometastasis are not uniform and vary from one case to another. In a salvage setting, the indication for surgery would be expanded. On the other hand, despite the lack of evidence, salvage surgery may be less indicated clinically for patients with obvious mediastinal lymph node metastases. It should be noted that salvage surgery after TKI treatment often requires a level of skill that is higher than simple partial resection, such as that in the present case. Local treatment should include not only surgery but also stereotactic radiotherapy, as suggested in the SINDAS trial. Overall, this case appears to have been treated well.

\section{Conclusions}

Multidisciplinary discussion with the participation of board certificated thoracic surgeon, oncologist, plays an irreplaceable role in the treatment of targeted therapy resistant advanced NSCLC patients. It is crucial important for clinicians to re-obtain molecular testing results for actionable or drug resistant biomarkers before initiating the subsequent therapy. CT guided percutaneous biopsy and fluid biopsy are effective methods to obtain samples for analysis. However, for patients who can tolerate surgery, salvage resection can not only provide enough sample for gene detection, but also prolong progression free survival. For resectable EGFR-TKI-resistant NSCLC patients, salvage surgery may be an option after MDT discussion. However, there is scarce evidence for the efficiency of the surgery after EGFR-TKI resistance is confirmed. Hence, further research is needed to clarify this issue.

\section{Acknowledgments}

Funding: Applied Basic Program of Department of Science and Technology of Sichuan Province, China (2018JY0593) (to Dr. HL).

\section{Footnote}

Reporting Checklist: The authors have completed the CARE reporting checklist. Available at https://dx.doi. org/10.21037/jtd-21-171

Conflicts of Interest: All authors have completed the ICMJE uniform disclosure form (available at https://dx.doi. org/10.21037/jtd-21-171). Dr. HH reports grants and personal fees from MSD, Lilly, AstraZeneca, BMS, Janssen, Ono and Chugai; personal fees from Merck Biophama, Kyowa-Kirin, Nihonkayaku and grants from DaiichiSankyo, Abbvie and Genomic health, outside the submitted work. The authors have no other conflicts of interests to declare.

Ethical Statement: The authors are responsible for ensuring the reliability and integrity of this case report and that issues related to this work have been appropriately investigated and resolved. Additionally, written informed consent was obtained from the patient and her family members for publication of this case report and all the affiliated images. All procedures performed in studies involving human participants were in accordance with the ethical standards of the institutional and/or national research committee(s) and with the Helsinki Declaration (as revised in 2013).

Open Access Statement: This is an Open Access article distributed in accordance with the Creative Commons Attribution-NonCommercial-NoDerivs 4.0 International License (CC BY-NC-ND 4.0), which permits the noncommercial replication and distribution of the article with the strict proviso that no changes or edits are made and the original work is properly cited (including links to both the formal publication through the relevant DOI and the license). See: https://creativecommons.org/licenses/by-nc-nd/4.0/.

\section{References}

1. Kobayashi K, Hagiwara K. Epidermal growth factor receptor (EGFR) mutation and personalized therapy in advanced nonsmall cell lung cancer (NSCLC). Target Oncol 2013;8:27-33.

2. Chen HF, Lei L, Wu LX, et al. Effect of icotinib on advanced lung adenocarcinoma patients with sensitive EGFR mutation detected in ctDNA by ddPCR. Transl Cancer Res 2019;8:2858-63.

3. Thunnissen E, van der Oord K, den Bakker M. Prognostic and predictive biomarkers in lung cancer. A review. Virchows Arch 2014;464:347-58.

4. Ma Y, Xin S, Lin Q, et al. The analysis of pharmacokinetic and pharmacogenomic impact on gefitinib efficacy in advanced non-small cell lung cancer patients: results from a prospective cohort study. Ann Transl Med 2019;7:806.

5. Yang Z, Hackshaw A, Feng Q, et al. Comparison of gefitinib, erlotinib and afatinib in non-small cell lung cancer: A meta-analysis. Int J Cancer 2017;140:2805-19. 
6. Santarpia M, Menis J, Chaib I, et al. Dacomitinib for the first-line treatment of patients with EGFR-mutated metastatic non-small cell lung cancer. Expert Rev Clin Pharmacol 2019;12:831-40.

7. Xiang X, Yu J, Lai Y, et al. L858R-positive lung adenocarcinoma with KRAS G12V, EGFR T790M and EGFR L858R mutations: A case report. Oncol Lett 2015;10:1293-6.

8. Zhong WZ, Chen KN, Chen C, et al. Erlotinib Versus Gemcitabine Plus Cisplatin as Neoadjuvant Treatment of Stage IIIA-N2 EGFR-Mutant Non-Small-Cell Lung Cancer (EMERGING-CTONG 1103): A Randomized Phase II Study. J Clin Oncol 2019;37:2235-45.

9. Wu YL, Zhou C, Liam CK, et al. First-line erlotinib versus gemcitabine/cisplatin in patients with advanced EGFR mutation-positive non-small-cell lung cancer: analyses from the phase III, randomized, open-label, ENSURE study. Ann Oncol 2015;26:1883-9.

10. Jung HA, Woo SY, Lee SH, et al. The different central nervous system efficacy among gefitinib, erlotinib and afatinib in patients with epidermal growth factor receptor mutation-positive non-small cell lung cancer. Transl Lung Cancer Res 2020;9:1749-58.

11. Zhou C, Wu YL, Chen G, et al. Erlotinib versus chemotherapy as first-line treatment for patients with advanced EGFR mutation-positive non-small-cell lung cancer (OPTIMAL, CTONG-0802): a multicentre, open-label, randomised, phase 3 study. Lancet Oncol 2011;12:735-42.

Cite this article as: Zang J, Horinouchi H, Hanaoka J, Funai K, Sakakura N, Liao H. The role of salvage surgery in the treatment of a gefitinib-resistant non-small cell lung cancer patient: a case report. J Thorac Dis 2021;13(7):45544559. doi: $10.21037 /$ jtd-21-171
12. Goto K, Nishio M, Yamamoto N, et al. A prospective, phase II, open-label study (JO22903) of first-line erlotinib in Japanese patients with epidermal growth factor receptor (EGFR) mutation-positive advanced non-small-cell lung cancer (NSCLC). Lung Cancer 2013;82:109-14.

13. Rosell R, Carcereny E, Gervais R, et al. Erlotinib versus standard chemotherapy as first-line treatment for European patients with advanced EGFR mutation-positive non-small-cell lung cancer (EURTAC): a multicentre, open-label, randomised phase 3 trial. Lancet Oncol 2012;13:239-46.

14. Zhong WZ, Wang Q, Mao WM, et al. Gefitinib versus vinorelbine plus cisplatin as adjuvant treatment for stage II-IIIA (N1-N2) EGFR-mutant NSCLC (ADJUVANT/ CTONG1104): a randomised, open-label, phase 3 study. Lancet Oncol 2018;19:139-48.

15. Raphael J, Vincent M, Boldt G, et al. Adjuvant Epidermal Growth Factor Receptor Tyrosine Kinase Inhibitors (TKIs) in Resected Non-Small Cell Lung Cancer (NSCLC): A Systematic Review and Meta-analysis. Am J Clin Oncol 2019;42:440-5.

16. Mok TS, Wu YL, Ahn MJ, et al. Osimertinib or PlatinumPemetrexed in EGFR T790M-Positive Lung Cancer. N Engl J Med 2017;376:629-40.

17. Takamochi K, Suzuki K, Sugimura H, et al. Surgical resection after gefitinib treatment in patients with lung adenocarcinoma harboring epidermal growth factor receptor gene mutation. Lung Cancer 2007;58:149-55. 